\title{
Controller Design for Descriptor-type Systems with Distributed Time- delay Using Extension
}

\author{
ALTUG IFTAR \\ Department of Electrical and Electronics Engineering \\ Eskisehir Technical University \\ 26555 Eskisehir, TURKEY
}

\begin{abstract}
Controller design using extension is considered for descriptor-type systems with distributed time-delay. The approach is useful in decentralized controller design using overlapping decompositions. In this approach, a given largescale system is decomposed overlappingly into a number of subsystems and expanded such that the overlapping parts appear as disjoint. Since the subsystems appear as disjoint for the expanded system, it is easier to design a descentralized controller for the expanded system. This controller is then contracted for implementation on the original system. In the present paper, it is shown how to contract such a controller to guarantee stability and desired performance for the original system.
\end{abstract}

Key Words: Systems, Systems Theory, Control, Time-Delay Systems

Received: April 23, 2020. Revised: January 4, 2021. Accepted: January 18, 2021. Published: February 2, 2021.

\section{Introduction}

A common way to attack the problem of analysis of and/or controller design for a large-scale system is to first decompose such a system into smaller subsystems [1]. However, many typical large-scale systems may have an overlapping part through which subsystems are interconnected [2]. For such a system a disjoint decomposition may not be useful. The overlapping decompositions approach has first been proposed in [3] and has been shown to be useful for such systems (e.g., [4]-[11]). Overlapping decompositions approach is based on the principle of inclusion [12]. A special case of inclusion, which is especially useful in controller design is extension, which was first introduced in [13] for finitedimensional systems.

Many physical systems, especially large-scale systems, may involve time-delays [14]. Such systems are usually named as time-delay systems [15]. These systems are infinitedimensional, since their state can not be represented by a finite number of state variables [16]. The dynamics of many time-delay systems can be described by delay-differential equations [17]. In such systems, if the delay-differential equations do not involve delayed versions of the derivative of the state vector, then these systems are said to be retarded. Otherwise, they are said to be neutral [18]. It is more difficult to control neutral systems, in general, compared to retarded systems, because neutral systems may contain chains of infinitely many modes going to infinity along vertical axes [19]. Retarded systems, however, although also exhibit infinitely many modes, can have only finitely many modes in any given right-half conplex-plane [20]. In fact, neutral time-delay systems is a more general class than retarded time-delay systems.

Delay-differential equations alone, however, may not suffice to describe the dynamics of some time-delay systems. In some cases, such delay-differential equations may need to be coupled with some delay-algebraic equations. Such systems are usually termed as descriptor-type systems [21]. Descriptor-type time-delay systems are inherently neutral, whether or not the equations describing their dynamics involve delayed versions of the derivative of the state vector. Dealing with descriptor-type time-delay systems is, in general, more challenging than dealing with retarded or nondescriptor-type neutral systems, because their response is, in general, discontinuous and may be impulsive [21].

Time-delays in a time-delay system can be discrete or distributed [22]. Systems with distributed time-delay can be named as distributed-time-delay systems. In the present work, we consider large-scale linear time-invariant (LTI) descriptor-type distributed-time-delay systems.

Although, as mentioned in the first paragraph above, there has been many works on the decentralized controller design using overlapping decompositions for finite-dimensional systems, such an approach has been considered for timedelay systems only recently. In particular, the principle of extension has recently been defined for retarded distributedtime-delay systems in [23] and for non-descriptor-type neutral distributed-time-delay systems in [24]. This principle has then been extended to descriptor-type distributed-time-delay systems in [25]. However, controller design was not considered in [25]. In the present work, we consider controller design using extension for descriptor-type distributed-timedelay systems. First, in Section II, we summarize the results of [25]. Then, in Section III, we discuss contractibility of controllers. We also show that if the controller designed for the expanded system stabilizes the expanded system and satisfies a performance criterion, then the contracted controller stabilizes the original system and satisfies a corresponding performance criterion.

Throughout the paper, $\mathbf{R}$ and $\mathbf{R}_{+}$denote the sets of, respectively, real numbers and non-negative real numbers. For positive integers $k$ and $l, \mathbf{R}^{k}$ and $\mathbf{R}^{k \times l}$ denote the spaces of, respectively, $k$-dimensional real vectors and $k \times l$ - 
dimensional real matrices. For $x \in \mathbf{R}^{k},\|x\|$ is the 2norm of $x$ and, for $X \in \mathbf{R}^{k \times l},\|X\|$ is the induced 2norm (i.e., the maximum singular value) of $X . I_{k}$ denotes the $k \times k$-dimensional identity matrix and 0 may denote a zero matrix of appropriate dimensions or a matrix function which is identically zero. Finally, bdiag $(\cdots)$ denotes a block diagonal matrix with indicated blocks on the main diagonal and $\operatorname{rank}(\cdot)$ denotes the rank of the indicated matrix.

For $M_{0} \in \mathbf{R}^{n \times p}$ and $M(\cdot):[-\tau, 0] \rightarrow \mathbf{R}^{n \times r}$, we say that $\left[\begin{array}{cc}M_{0} & M(\cdot)\end{array}\right]$ has full row-rank, if for any $\xi \in \mathbf{R}^{n}$, there exist $u_{0} \in \mathbf{R}^{p}$ and $\varphi:[-\tau, 0] \rightarrow \mathbf{R}^{r}$ such that $\xi=$ $M_{0} u_{0}+\int_{-\tau}^{0} M(\theta) \varphi(\theta) d \theta$.

\section{Extension Principle}

In this section, we summarize the results of [25]. Consider two LTI descriptor-type distributed-time-delay systems, $\Sigma$ :

$$
\begin{gathered}
E_{0} \dot{x}(t)=A_{0} x(t)+B_{0} u(t)+\int_{-\tau}^{0}(A(\theta) x(t+\theta) \\
+B(\theta) u(t+\theta)) d \theta \\
y(t)=C_{0} x(t)+\int_{-\tau}^{0} C(\theta) x(t+\theta) d \theta
\end{gathered}
$$

and $\hat{\Sigma}$ :

$$
\begin{gathered}
\hat{E}_{0} \dot{\hat{x}}(t)=\hat{A}_{0} \hat{x}(t)+\hat{B}_{0} \hat{u}(t)+\int_{-\tau}^{0}(\hat{A}(\theta) \hat{x}(t+\theta) \\
+\hat{B}(\theta) \hat{u}(t+\theta)) d \theta \\
\hat{y}(t)=\hat{C}_{0} \hat{x}(t)+\int_{-\tau}^{0} \hat{C}(\theta) \hat{x}(t+\theta) d \theta
\end{gathered}
$$

where $x(t) \in \mathbf{R}^{n}$ and $\hat{x}(t) \in \mathbf{R}^{\hat{n}}$ are the state, $u(t) \in \mathbf{R}^{p}$ and $\hat{u}(t) \in \mathbf{R}^{\hat{p}}$ are the input, and $y(t) \in \mathbf{R}^{q}$ and $\hat{y}(t) \in \mathbf{R}^{\hat{q}}$ are the output vectors of, respectively, $\Sigma$ and $\hat{\Sigma}$ at time $t$. $\tau>0$ is the maximum time-delay in $\Sigma$ and $\hat{\Sigma}$. It is assumed that the dimensions of the state, input, and output vectors of $\hat{\Sigma}$ are greater than or equal to those of $\Sigma$; i.e., $\hat{n} \geq n, \hat{p} \geq p$, and $\hat{q} \geq q$. It is also assumed that $E_{0} \in \mathbf{R}^{n \times n}, A_{0} \in \mathbf{R}^{n \times n}$, $B_{0} \in \mathbf{R}^{n \times p}, C_{0} \in \mathbf{R}^{q \times n}, \hat{E}_{0} \in \mathbf{R}^{\hat{n} \times \hat{n}}, \hat{A}_{0} \in \mathbf{R}^{\hat{n} \times \hat{n}}, \hat{B}_{0} \in$ $\mathbf{R}^{\hat{n} \times \hat{p}}$, and $\hat{C}_{0} \in \mathbf{R}^{\hat{q} \times \hat{n}}$ are constant matrices and $A(\cdot)$ : $[-\tau, 0] \rightarrow \mathbf{R}^{n \times n}, B(\cdot):[-\tau, 0] \rightarrow \mathbf{R}^{n \times p}, C(\cdot):[-\tau, 0] \rightarrow$ $\mathbf{R}^{q \times n}, \hat{A}(\cdot):[-\tau, 0] \rightarrow \mathbf{R}^{\hat{n} \times \hat{n}}, \hat{B}(\cdot):[-\tau, 0] \rightarrow \mathbf{R}^{\hat{n} \times \hat{p}}$, and $\hat{C}(\cdot):[-\tau, 0] \rightarrow \mathbf{R}^{\hat{q} \times \hat{n}}$ are bounded matrix functions. It is further assumed that $\operatorname{rank}\left(E_{0}\right)=n_{e}<n$ and $\operatorname{rank}\left(\hat{E}_{0}\right)=$ $\hat{n}_{e}<\hat{n}$, which make both systems $\Sigma$ and $\hat{\Sigma}$ descriptor-type [21]. For the existence and uniqueness of solutions, however, we assume the following:

Assumption 1: $\operatorname{rank}\left(\mathcal{U} A_{0} \mathcal{V}\right)=n-n_{e}$, where the rows of $\mathcal{U}$ (respectively, columns of $\mathcal{V}$ ) span the left (respectively, right) null space of $E_{0}$.

Assumption 2: $\operatorname{rank}\left(\hat{\mathcal{U}} \hat{A}_{0} \hat{\mathcal{V}}\right)=\hat{n}-\hat{n}_{e}$, where the rows of $\hat{\mathcal{U}}$ (respectively, columns of $\hat{\mathcal{V}}$ ) span the left (respectively, right) null space of $\hat{E}_{0}$.

These two assumptions guarantee the existence and uniqueness of solutions to (1) and (3), respectively, under suitable initial conditions [17], which are assumed to be given as:

$$
x(\theta)=\phi(\theta) \quad \text { and } \quad \hat{x}(\theta)=\hat{\phi}(\theta), \quad \theta \in[-\tau, 0],
$$

for some well-defined functions $\phi:[-\tau, 0] \rightarrow \mathbf{R}^{n}$ and $\hat{\phi}$ : $[-\tau, 0] \rightarrow \mathbf{R}^{\hat{n}}$, respectively.

We note that, under Assumptions 1 and 2, by a suitable state transformation, the delay-free parts of (1) and (3) can be decoupled into their differential and algebraic parts [21] (there will still be coupling through delayed dynamics, in general, however). That is, by using a state trasformation, (1)-(4) can be put into a form where

$$
E_{0}=\operatorname{bdiag}\left(I_{n_{e}}, 0\right) \text { and } A_{0}=\operatorname{bdiag}\left(A_{0}^{1}, I_{n-n_{e}}\right)
$$

and

$$
\hat{E}_{0}=\operatorname{bdiag}\left(I_{\hat{n}_{e}}, 0\right) \quad \text { and } \quad \hat{A}_{0}=\operatorname{bdiag}\left(\hat{A}_{0}^{1}, I_{\hat{n}-\hat{n}_{e}}\right)
$$

Although, in such a form, the equations may look appealing, the states may lose their physical meaning. Furthermore, some matrices/matrix functions may become ill-conditioned. Therefore, except in the only if part of Theorem 1 below, we assume that (6) and (7) may not hold in general.

The principle of extension was defined in [25] as follows:

Definition 1: $\hat{\Sigma}$ is said to be an extension of $\Sigma$ if there exist full-rank matrices

$$
T \in \mathbf{R}^{\hat{n} \times n}, \quad R \in \mathbf{R}^{p \times \hat{p}}, \quad \text { and } \quad S \in \mathbf{R}^{\hat{q} \times q}
$$

such that for all $\phi(\cdot)$ and for all $\hat{u}(\cdot)$, the choice

$$
\hat{\phi}(\theta)=T \phi(\theta), \quad \theta \in[-\tau, 0]
$$

and

$$
u(t)=R \hat{u}(t), \quad t \geq-\tau
$$

implies

$$
\hat{x}(t)=T x(t), \quad t \geq-\tau
$$

and

$$
\hat{y}(t)=S y(t), \quad t \geq 0
$$

Extension defined above is a generalization of extension, which was first defined for finite-dimensional systems in [13]. In the case of finite-dimensional systems, the advantage of using extension rather than the more general principle of inclusion is that, if extension is used then any controller designed for the expanded system can be contracted for implemetation on the original system [26]. We will show that (see Corollary 2 below) the same is also true in the present case.

The necessary and sufficient conditions for $\hat{\Sigma}$ to be an extension of $\Sigma$ are given by the following theorem, which was first presented in [25].

Theorem 1: $\hat{\Sigma}$ is an extension of $\Sigma$ if there exist full-rank matrices as in (8) such that

$$
\hat{E}_{0} T=T E_{0},
$$




$$
\begin{aligned}
& \hat{A}_{0} T=T A_{0} \text { and } \hat{A}(\theta) T=T A(\theta), \forall \theta \in[-\tau, 0], \\
& \hat{B}_{0}=T B_{0} R \text { and } \hat{B}(\theta)=T B(\theta) R, \forall \theta \in[-\tau, 0],
\end{aligned}
$$

and

$$
\hat{C}_{0} T=S C_{0} \text { and } \hat{C}(\theta) T=S C(\theta), \forall \theta \in[-\tau, 0] .
$$

Furthermore, the above conditions are also necessary if (1)(4) are written in a form such that (6) and (7) hold and that $\left[\begin{array}{lll}B_{0}^{2} & A^{2}(\cdot) & B^{2}(\cdot)\end{array}\right]$ has full row-rank, where $B_{0}^{2}, A^{2}(\cdot)$, and $B^{2}(\cdot)$ denote the last $n-n_{e}$ rows of $B_{0}, A(\cdot)$, and $B(\cdot)$, respectively.

Proof: See [25].

Remark: As it was also remarked in [25], (6) and (7) are used to prove only the necessity of (13) and the first conditions in (14) and (16). Given that (13) and the first condition in (14) hold, (15) and the second conditions in (14) and (16) are necessary, whether or not (6) and (7) hold. Furthermore, the assumption that $\left[\begin{array}{lll}B_{0}^{2} & A^{2}(\cdot) & B^{2}(\cdot)\end{array}\right]$ has full row-rank is used only to prove the necessity of the first condition in (16). Thus, even if this assumption does not hold, provided that (13) and the first condition in (14) hold, (13)-(15), as well as the second condition in (16) are still necessary. Moreover, as mentioned above, (1)-(4) can always be written in a form such that (6) and (7) hold. In this form, if $\left[\begin{array}{ccc}B_{0}^{2} & A^{2}(\cdot) & B^{2}(\cdot)\end{array}\right]$ does not have full row-rank, it means that $x(t)$, for $t>0$, can take values only in a subspace of $\mathbf{R}^{n}$ (and if $\hat{\Sigma}$ is an extension of $\Sigma, \hat{x}(t)$, for $t>0$, can take values only in a subspace of $\mathbf{R}^{\hat{n}}$ ). More precisely, $x_{2}(t)$, for $t>0$, can take values only in the range space of $\left[\begin{array}{lll}B_{0}^{2} & A^{2}(\cdot) & B^{2}(\cdot)\end{array}\right]$ and $\hat{x}_{2}(t)$, for $t>0$, can take values only in the range space of $\left[\begin{array}{lll}\hat{B}_{0}^{2} & \hat{A}^{2}(\cdot) & \hat{B}^{2}(\cdot)\end{array}\right]$, where $\hat{B}_{0}^{2}, \hat{A}^{2}(\cdot)$, and $\hat{B}^{2}(\cdot)$ denote the last $\hat{n}-\hat{n}_{e}$ rows of $\hat{B}_{0}, \hat{A}(\cdot)$, and $\hat{B}(\cdot)$, respectively. Thus, in such a case, by a state transformation, the dimensions of both $x(t)$ and $\hat{x}(t)$ can be reduced so that for the new state equations the assumption that $\left[\begin{array}{ccc}B_{0}^{2} & A^{2}(\cdot) & B^{2}(\cdot)\end{array}\right]$ has full row-rank is satisfied.

As it was also indicated in [25], without any loss of generality, the matrices and the matrix functions of $\Sigma$ and $\hat{\Sigma}$ can be related as follows:

$$
\begin{gathered}
\hat{E}_{0}=T E_{0} \tilde{T}+M_{E}^{0}, \quad \hat{A}_{0}=T A_{0} \tilde{T}+M_{A}^{0}, \\
\hat{B}_{0}=T B_{0} R+M_{B}^{0}, \quad \hat{C}_{0}=S C_{0} \tilde{T}+M_{C}^{0} \\
\hat{A}(\theta)=T A(\theta) \tilde{T}+M_{A}(\theta), \quad \theta \in[-\tau, 0], \\
\hat{B}(\theta)=T B(\theta) R+M_{B}(\theta), \quad \theta \in[-\tau, 0],
\end{gathered}
$$

and

$$
\hat{C}(\theta)=S C(\theta) \tilde{T}+M_{C}(\theta), \quad \theta \in[-\tau, 0],
$$

where $T, R$, and $S$ are the matrices introduced in (8) and $\tilde{T} \in \mathbf{R}^{n \times \hat{n}}$ is a left-inverse of $T$, satisfying $\tilde{T} T=$ $I_{n}$ (such $\tilde{T}$ exists, since $T$ is of full-rank and $\hat{n} \geq n$ ). Furthermore, $M_{E}^{0} \in \mathbf{R}^{\hat{n} \times \hat{n}}, M_{A}^{0} \in \mathbf{R}^{\hat{n} \times \hat{n}}, M_{B}^{0} \in \mathbf{R}^{\hat{n} \times \hat{p}}$, and $M_{C}^{0} \in \mathbf{R}^{\hat{q} \times \hat{n}}$ are the so-called complementary matrices and $M_{A}(\cdot):[-\tau, 0] \rightarrow \mathbf{R}^{\hat{n} \times \hat{n}}, M_{B}(\cdot):[-\tau, 0] \rightarrow \mathbf{R}^{\hat{n} \times \hat{p}}$, and
$M_{C}(\cdot):[-\tau, 0] \rightarrow \mathbf{R}^{\hat{q} \times \hat{n}}$ are the so-called complementary matrix functions, which are bounded matrix functions. As we will see in Section IV, this representation facilitates defining an expansion of an overlappingly decomposed system. The necessary and sufficient conditions for $\hat{\Sigma}$ to be an extension of $\Sigma$ can equivalently be stated in terms of these complementary matrices and matrix functions [25]:

Corollary 1: $\hat{\Sigma}$ is an extension of $\Sigma$ if

$$
\begin{gathered}
M_{E}^{0} T=0, \\
M_{A}^{0} T=0 \quad \text { and } \quad M_{A}(\theta) T=0, \forall \theta \in[-\tau, 0], \\
M_{B}^{0}=0 \quad \text { and } \quad M_{B}(\theta)=0, \forall \theta \in[-\tau, 0],
\end{gathered}
$$

and

$$
M_{C}^{0} T=0 \text { and } M_{C}(\theta) T=0, \forall \theta \in[-\tau, 0] .
$$

Furthermore, the above conditions are also necessary if (1)(4) are written in a form such that (6) and (7) hold and that $\left[\begin{array}{lll}B_{0}^{2} & A^{2}(\cdot) & B^{2}(\cdot)\end{array}\right]$ has full row-rank.

Proof: See [25].

\section{Contractibility of Controllers}

In the approach of overlapping decompositions, a system is first overlappingly decomposed and then expanded so that overlapping parts appear as disjoint. A controller is then designed for this expanded system and then contracted for implementation on the original system. In order for the contracted controller work on the original system, however, it must be contractible. Therefore, in this section we will consider contractibility of controllers.

In [27], it was shown that a LTI retarded time-delay system can be stabilized by a centralized LTI retarded time-delay controller if and only if it can be stabilized by a LTI finitedimensional centralized controller. This result was generalized to possibly descriptor-type neutral time-delay systems under possibly decentralized control in [28]. Specifically, it was shown in [28] that a possibly descriptor-type LTI neutral time-delay system can be stabilized by a possibly descriptortype LTI (de)centralized neutral time-delay controller if and only if it can be stabilized by a LTI (de)centralized finitedimensional controller. Therefore, in here, we will consider only finite-dimensional controllers. Thus, for $\Sigma$, we consider a controller, to be denoted by $\Gamma$, of the form

$$
\begin{aligned}
& \dot{z}(t)=F z(t)+G w(t) \\
& v(t)=H z(t)+K w(t)
\end{aligned}
$$

and, for $\hat{\Sigma}$, we consider a controller, to be denoted by $\hat{\Gamma}$, of the form

$$
\begin{aligned}
& \dot{\hat{z}}(t)=\hat{F} \hat{z}(t)+\hat{G} \hat{w}(t) \\
& \hat{v}(t)=\hat{H} \hat{z}(t)+\hat{K} \hat{w}(t)
\end{aligned}
$$

Here, $z(t) \in \mathbf{R}^{m}$ and $\hat{z}(t) \in \mathbf{R}^{\hat{m}}$ are the state, $w(t) \in \mathbf{R}^{q}$ and $\hat{w}(t) \in \mathbf{R}^{\hat{q}}$ are the input, and $v(t) \in \mathbf{R}^{p}$ and $\hat{v}(t) \in$ $\mathbf{R}^{\hat{p}}$ are the output vectors of, respectively, $\Gamma$ and $\hat{\Gamma}$ at time $t$. Furthermore, $F \in \mathbf{R}^{m \times m}, G \in \mathbf{R}^{m \times q}, H \in \mathbf{R}^{p \times m}$, 
$K \in \mathbf{R}^{p \times q}, \hat{F} \in \mathbf{R}^{\hat{m} \times \hat{m}}, \hat{G} \in \mathbf{R}^{\hat{m} \times \hat{q}}, \hat{H} \in \mathbf{R}^{\hat{p} \times \hat{m}}$, and $\hat{K} \in \mathbf{R}^{\hat{p} \times \hat{q}}$ are constant matrices. The initial conditions for $\Gamma$ and $\hat{\Gamma}$ are assumed to be given as:

$$
z(0)=\zeta_{0} \quad \text { and } \quad \hat{z}(0)=\hat{\zeta}_{0},
$$

for some $\zeta_{0} \in \mathbf{R}^{m}$ and $\hat{\zeta}_{0} \in \mathbf{R}^{\hat{m}}$, respectively.

The controllers $\Gamma$ and $\hat{\Gamma}$ are to be applied to $\Sigma$ and $\hat{\Sigma}$, respectively, by letting

$$
w(t)=y(t)-r(t) \quad \text { and } \quad \hat{w}(t)=\hat{y}(t)-\hat{r}(t)
$$

and

$$
u(t)=v(t)+d(t) \quad \text { and } \quad \hat{u}(t)=\hat{v}(t)+\hat{d}(t)
$$

for $t \geq 0$, where $r(t) \in \mathbf{R}^{q}$ and $d(t) \in \mathbf{R}^{p}$ are some external inputs (possibly a reference and a disturbance, respectively) at time $t$, for $\Sigma_{c}$, where $\Sigma_{c}$ denotes the closed-loop system obtained by applying $\Gamma$ to $\Sigma$, and $\hat{r}(t) \in \mathbf{R}^{\hat{q}}$ and $\hat{d}(t) \in \mathbf{R}^{\hat{p}}$ are some external inputs at time $t$, for $\hat{\Sigma}_{c}$, where $\hat{\Sigma}_{c}$ denotes the closed-loop system obtained by applying $\hat{\Gamma}$ to $\hat{\Sigma}$.

We note that, although the maximum time-delay in the closed-loop systems will be $2 \tau$, due to the assumption that the loops are closed at time $t=0$, only $x(t)$ for $t \geq-\tau$ will affect $\Gamma$ and only $z(t)$ for $t \geq 0$ will affect $\Sigma$ (similarly for $\hat{\Gamma}$ and $\hat{\Sigma}$ ). Thus, (5) and (30) will also define the initial conditions of the closed-loop systems.

We can now define contractibility:

Definition 2: Suppose that the connection in (31) is made but the connection in (32) is not made. The controller $\hat{\Gamma}$ for $\hat{\Sigma}$ is said to be contractible to the controller $\Gamma$ for $\Sigma$ if there exist full-rank matrices as in (8) and a full row-rank matrix $P \in \mathbf{R}^{m \times \hat{m}}$ such that for all initial conditions $\phi(\cdot)$ of $\Sigma$, for all inputs $\hat{u}(\cdot)$ of $\hat{\Sigma}$, for all external inputs $r(\cdot)$ of $\Sigma_{c}$, and for all initial conditions $\hat{\zeta}_{0}$ of $\hat{\Gamma}$, the choice (9), (10),

$$
\zeta_{0}=P \hat{\zeta}_{0}
$$

and

$$
\hat{r}(t)=S r(t), \quad t \geq 0
$$

implies

$$
z(t)=P \hat{z}(t), \quad t \geq 0
$$

and

$$
v(t)=R \hat{v}(t), \quad t \geq 0
$$

Note that, the existence of a full row-rank matrix $P \in$ $\mathbf{R}^{m \times \hat{m}}$, in particular requires $\hat{m} \geq m$. However, this is natural, since $\Sigma$, in general, forms a part of $\hat{\Sigma}$, and hence, should not require a controller with a larger dimensional state vector [23]. Contractibility is needed so that the condition (10) is satisfied after the application of the controllers. Although, however, (10) is required for $t \geq-\tau$, (36) is required only for $t \geq 0$, since the controllers are to be applied starting at time $t=0$.

The conditions for $\hat{\Gamma}$ to be contractible to $\Gamma$ are given by the following theorem:
Theorem 2: Suppose that $\hat{\Sigma}$ is an extension of $\Sigma$. Then, the controller $\hat{\Gamma}$ for $\hat{\Sigma}$ is contractible to the controller $\Gamma$ for $\Sigma$ if there exists a full row-rank matrix $P \in \mathbf{R}^{m \times \hat{m}}$ such that

$$
\begin{gathered}
F P=P \hat{F}, \quad G=P \hat{G} S, \\
H P=R \hat{H}, \quad \text { and } K=R \hat{K} S,
\end{gathered}
$$

where $R$ and $S$ are as in (8).

Proof: Since $\hat{\Sigma}$ is an extension of $\Sigma$, the choice (9) and (10) implies (12). Then, (31) and (34) implies

$$
\hat{w}(t)=S w(t), \quad t \geq 0 .
$$

To establish (35), premultiply both sides of (28) by $P$ and use (39) to obtain

$$
P \dot{\hat{z}}(t)=P \hat{F} \hat{z}(t)+P \hat{G} S w(t)
$$

Next, use (37) in (40) and compare with (26). Together with (33), by the uniqueness of solutions, this establishes (35). Next, to establish (36), premultiply both sides of (29) by $R$ and use (39) and (38) to obtain

$$
R \hat{v}(t)=H P \hat{z}(t)+K w(t)
$$

Next, use (35) in the right-hand-side of (41) and compare with (27). This establishes (36), which concludes the proof.

Since a controller $\hat{\Gamma}$ is first to be designed for $\hat{\Sigma}$ and then to be contrated for implementation on $\Sigma$, it is important that any controller $\hat{\Gamma}$ for $\hat{\Sigma}$ to be contractible to a controller $\Gamma$ for $\Sigma$. This is in fact the case if $\hat{\Sigma}$ is an extension of $\Sigma$ :

Corollary 2: If $\hat{\Sigma}$ is an extension of $\Sigma$, then any controller $\hat{\Gamma}$ of the form (28)-(29) for $\hat{\Sigma}$ is contractible to a controller $\Gamma$ of the form (26)-(27) for $\Sigma$ with

$$
\begin{gathered}
F=\hat{F}, \quad G=\hat{G} S, \\
H=R \hat{H}, \quad \text { and } K=R \hat{K} S,
\end{gathered}
$$

where $R$ and $S$ are as in (8).

Proof: With $m=\hat{m}$ and $P=I_{m}$, (42)-(43) are equivalent to (37)-(38).

Now, suppose that $\Gamma$ is applied to $\Sigma$ and $\hat{\Gamma}$ is applied to $\hat{\Sigma}$ by making the connections in (31) and (32) starting at time $t=0$. Let us denote the closed-loop system obtained by applying $\Gamma$ to $\Sigma$ by $\Sigma_{c}$ and the closed-loop system obtained by applying $\hat{\Gamma}$ to $\hat{\Sigma}$ by $\hat{\Sigma}_{c}$. In [23], for the case of retarded distributed-time-delay systems, it was proved that when the expanded system is an extension of the original system and the controller for the expanded system is contractible to the controller for the original system, then certain stability and performance relations between the two closed-loop systems hold. For stability, we can use the usual definition of asymptotic or exponential stability for timedelay systems (e.g., see [29]). For performance, a specific tracking requirement was used in [23], which is defined as follows:

Definition 3: A closed-loop system, such as $\Sigma_{c}$, is said to achieve good tracking for references $r(\cdot)$, with respect to a 
tolerance function $g: \mathbf{R}_{+} \rightarrow \mathbf{R}_{+}$and a disturbance bound $f:[-\tau, \infty) \rightarrow \mathbf{R}_{+}$, if, assuming that the initial state is zero, the output, $y(\cdot)$, satisfies $\|y(t)-r(t)\| \leq g(t), \forall t \geq 0$, for all disturbances which satisfy $\|d(t)\| \leq f(t), \forall t \geq-\tau$.

Now, we can state the following results, the proofs of which follow the same lines as the proofs of the corresponding theorems in [23]:

Theorem 3: Suppose that $\hat{\Sigma}$ is an extension of $\Sigma, \hat{\Gamma}$ is contractible to $\Gamma$, and $\hat{\Gamma}$ stabilizes $\hat{\Sigma}$. Then, $\Gamma$ stabilizes $\Sigma$.

Theorem 4: Suppose that $\hat{\Sigma}$ is an extension of $\Sigma$ and $\hat{\Gamma}$ is contractible to $\Gamma$. Let $R$ and $S$ be as in Definition 1 and $\tilde{R}$ and $\tilde{S}$ be such that $R \tilde{R}=I_{p}$ and $\tilde{S} S=I_{q} \cdot{ }^{1}$ Suppose that $\hat{\Sigma}_{c}$ achieves good tracking for references $\hat{r}(t)=\operatorname{Sr}(t)$, $t \geq 0$, for some $r(\cdot)$, with respect to $\hat{g}: \mathbf{R}_{+} \rightarrow \mathbf{R}_{+}$and $\hat{f}:[-\tau, \infty) \rightarrow \mathbf{R}_{+}$. Then, $\Sigma_{c}$ achieves good tracking for references $r(\cdot)$, with respect to $g(\cdot):=\|\tilde{S}\| \hat{g}(\cdot)$ and $f(\cdot):=$ $\frac{1}{\|\tilde{R}\|} \hat{f}(\cdot)$.

\section{Conclusions}

Controller design using extension has been considered for descriptor-type systems with distributed time-delay. First, the principle of extension has been presented, as it was first introduced in [25]. Contractibility of controllers has then been discussed and it has been shown that if the expanded system is an extension of the original system, then any controller designed for the expanded system is contractible for implementation on the original system. Furthermore, it has also been shown that if the expanded closed-loop system is stable and achieves desired performance, then the original closed-loop system is also stable and achieves desired performance.

Although only descriptor-type distributed-time-delay systems described by delay-differential-algebraic equations, where the equations do not involve delayed versions of the derivative of the state vector has been considered, the results can be extended to systems, where the equations do involve delayed versions of the derivative of the state vector.

\section{References}

[1] D. D. Šiljak, Large-Scale Dynamic Systems: Stability and Structure. New York: North-Holland, 1978.

[2] — Decentralized Control of Complex Systems. San Diego: Academic Press, Inc., 1991.

[3] M. Ikeda and D. D. Šiljak, "Overlapping decompositions, expansions, and contractions of dynamic systems," Large Scale Systems, vol. 1, pp. 29-38, 1980

[4] M. Ikeda, D. D. Šiljak, and D. E. White, "Decentralized control with overlapping information sets," Journal of Optimization Theory and Applications, vol. 34, pp. 279-310, 1981.

[5] A. Iftar and F. Khorrami, "A comparison of multiple time-scale analysis and overlapping decomposition," IEEE Transactions on Systems, Man, and Cybernetics, vol. SMC-19, pp. 1296-1300, 1989.

${ }^{1}$ There exist such $\tilde{R}$ and $\tilde{S}$ since $R$ and $S$ are of full-rank and $\hat{p} \geq$ $p$ and $\hat{q} \geq q$. Note that it makes sense to choose these matrices as the Moore-Penrose inverses [30] of $R$ and $S$, respectively, so that they will have minimum norm, which will, in turn, allow minimum tolerance function $g$ and maximum disturbance bound $f$.
[6] A. İftar and Ü. Özgüner, "Overlapping decompositions, expansions, contractions, and stability of hybrid systems," IEEE Transactions on Automatic Control, vol. 43, pp. 1040-1055, 1998.

[7] Ü. Özgüner, C. Hatipoğlu, A. İftar, and K. Redmill, "Hybrid control design for a three vehicle scenario demonstration using overlapping decompositions," in Hybrid Systems IV, Lecture Notes in Computer Science, vol 1273, P. J. Antsaklis, Ed., Springer Verlag, 1997, pp. 294-328.

[8] S. S. Stanković, M. J. Stanojević, and D. D. Šiljak, "Decentralized overlapping control of a platoon of vehicles," IEEE Transactions on Control Systems Technology, vol. 8, pp. 816-832, 2000.

[9] B. Ataşlar and A. İtar, "Decentralized routing controller design using overlapping decompositions," International Journal of Control, vol. 72, pp. 1175-1192, 1999.

[10] A. Aybar and A. İftar, "Overlapping decompositions and expansions of Petri nets," IEEE Transactions on Automatic Control, vol. 47, pp. 511-515, 2002.

[11] A. Iftar, "Decentralized robust control based on overlapping decompositions," in Preprints of the 10th IFAC Symposium on Large Scale Systems, Osaka, Japan, Jul. 2004, pp. 605-609.

[12] M. Ikeda, D. D. Šiljak, and D. E. White, "An inclusion principle for dynamic systems," IEEE Transactions on Automatic Control, vol. AC29, pp. 244-249, 1984.

[13] A. İftar and Ü. Özgüner, "Contractible controller design and optimal control with state and input inclusion," Automatica, vol. 26, pp. 593597, 1990.

[14] L. Bakule, "Decentralized control: An overview," Annual Reviews in Control, vol. 32, pp. 87-98, 2008.

[15] J.-P. Richard, "Time-delay systems: an overview of some recent advances and open problems," Automatica, vol. 39, pp. 1667-1694, 2003

[16] C. Foias, H. Özbay, and A. Tannenbaum, Robust Control of Infinite Dimensional Systems: Frequency Domain Methods, Lecture Notes in Control and Information Sciences, No. 209. London: Springer-Verlag, 1996.

[17] J. K. Hale and S. M. Verduyn-Lunel, Introduction to Functional Differential Equations. New York: Springer-Verlag, 1993.

[18] S.-I. Niculescu, Delay Effects on Stability: A Robust Control Approach, Lecture Notes in Control and Information Sciences, No. 269. London: Springer-Verlag, 2001

[19] W. Michiels and S.-I. Niculescu, Stability and Stabilization of TimeDelay Systems. Philadelphia: SIAM, 2007.

[20] Z. Wu and W. Michiels, "Reliably computing all characteristic roots of delay differential equations in a given right half plane using a spectral method," Journal of Computational and Applied Mathematics, vol. 236, pp. 2499-2514, 2012.

[21] G.-R. Duan, Analysis and Design of Descriptor Linear Systems. New York: Springer, 2010

[22] K. L. Cooke and Z. Grossman, "Discrete delay, distributed delay and stability switches," Journal of Mathematical Analysis and Applications, vol. 86, pp. 592-627, 1982.

[23] A. İftar, "Extension principle and controller design for systems with distributed time-delay," Kybernetika, vol. 53, pp. 630-652, 2017.

[24] _ , "Controller design using extension for neutral distributed-timedelay systems," in Proceedings of the 9th International Conference on Information Technology and Electrical Engineering, Phuket, Thailand, Oct. 2017.

[25] _ "Extension principle for descriptor-type systems with distributed time-delay," in Proceedings of the 24th International Conference on System Theory, Control and Computing, Sinaia, Romania, Oct. 2020

[26] — - "Decentralized estimation and control with overlapping input, state, and output decomposition," Automatica, vol. 29, pp. 511-516, 1993.

[27] E. W. Kamen, P. P. Khargonekar, and A. Tannenbaum, "Stabilization of time-delay systems using finite-dimensional compensators," IEEE Transactions on Automatic Control, vol. AC-30, pp. 75-78, 1985.

[28] H. E. Erol and A. Ifftar, "Stabilization of decentralized descriptor-type neutral time-delay systems by time-delay controllers," Automatica, vol. 64, pp. 262-269, 2016.

[29] W. Michiels and S.-I. Niculescu, Stability, Control, and Computation for Time-Delay Systems: An Eigenvalue-Based Approach. Philadelphia: SIAM, 2014.

[30] R. Penrose and J. A. Todd, "On best approximate solutions of linear matrix equations," Mathematical Proceedings of the Cambridge Philosophical Society, vol. 52, pp. 17-19, 1956. 
Contribution of Individual Authors to the Creation of a Scientific Article (Ghostwriting Policy)

The author contributed in the present research, at all stages from the formulation of the problem to the final findings and solution.

Sources of Funding for Research Presented in a Scientific Article or Scientific Article Itself

No funding was received for conducting this study.

\section{Conflict of Interest}

The author has no conflict of interest to declare that is relevant to the content of this article.

Creative Commons Attribution License 4.0 (Attribution 4.0 International, CC BY 4.0)

This article is published under the terms of the Creative Commons Attribution License 4.0

https://creativecommons.org/licenses/by/4.0/deed.en US 